\title{
Listening to music and physiological and psychological functioning: The mediating role of emotion regulation and stress reactivity
}

\author{
M.V. Thoma ${ }^{\mathrm{a}}$, U. Scholz ${ }^{\mathrm{b}}$, U. Ehlert ${ }^{\mathrm{c}}$ and U.M. Nater ${ }^{\mathrm{d} *}$ \\ ${ }^{a}$ Department of Psychology, Brandeis University, Brown 129, MS 062, 415 South Street, \\ Waltham, MA 02453 2728, USA; ${ }^{b}$ Social and Health Psychology, University of Zurich, \\ Binzmuehlestrasse 14, Box 26, Zurich 8050, Switzerland; ${ }^{C}$ Clinical Psychology and \\ Psychotherapy, University of Zurich, Binzmuehlestrasse 14, Box 26, Zurich 8050, \\ Switzerland; 'Clinical Biopsychology, University of Marburg, Gutenbergstrasse 18, \\ Marburg 35032, Germany
}

\begin{abstract}
Music listening has been suggested to have short term beneficial effects. The aim of this study was to investigate the association and potential mediating mechanisms between various aspects of habitual music listening behaviour and physiological and psychological functioning. An internet based survey was conducted in university students, measuring habitual music listening behaviour, emotion regulation, stress reactivity, as well as physiological and psychological functioning. A total of 1230 individuals (mean $24.89 \pm 5.34$ years, $55.3 \%$ women) completed the questionnaire. Quantitative aspects of habitual music listening behaviour, i.e. average duration of music listening and subjective relevance of music, were not associated with physiological and psychological functioning. In contrast, qualitative aspects, i.e. reasons for listening (especially 'reducing loneliness and aggression', and 'arousing or intensifying specific emotions') were significantly related to physiological and psychological functioning (all $p$ 0.001). These direct effects were mediated by distress augmenting emotion regulation and individual stress reactivity. The habitual music listening behaviour appears to be a multifaceted behaviour that is further influenced by dispositions that are usually not related to music listening. Consequently, habitual music listening behaviour is not obviously linked to physiological and psychological functioning.
\end{abstract}

Keywords: music; physiological and psychological functioning; distress augmenting emotion regulation; individual stress reactivity

\section{Introduction}

The development of effective stress prevention or management has become an important endeavour of current research efforts in health psychology. In particular, cost-efficient interventions, such as music therapy, have received special interest in terms of coping with stress and stress-related health issues. The potential of music to trigger intensive emotions (Blood \& Zatorre, 2001) and to attenuate psychophysiological activation (Khalfa, Bella, Roy, Peretz, \& Lupien, 2003; Nater, Abbruzzese,

*Corresponding author. Email: nater@uni marburg.de 
Krebs, \& Ehlert, 2006) might be the main reason for its beneficial effect and, consequently, for its increased therapeutic use in health care (see reviews in Evans, 2002; Nilsson, 2008).

Previous research has investigated the beneficial effect of listening to music in the context of medical settings (Ebneshahidi \& Mohseni, 2008; Pothoulaki et al., 2008; Voss et al., 2004) and in laboratory-based studies (Khalfa et al., 2003; Roy, Peretz, \& Rainville, 2007). These studies found that listening to music has an acute, i.e. short-term beneficial effect on various psychological and physiological parameters in the listener. In addition, these short-term effects seem to vary depending on the type of music (Burns et al., 2002; Roy et al., 2007).

Listening to music on a regular basis can be understood as habituation to the possible effects of music. From this perspective, the question arises of whether shortterm beneficial effects accumulate over time, i.e. are there any long-term beneficial effects of listening to music? Long-term effects have mainly been studied in the context of formal music training and potential enhancement of non-musical aspects of cognitive development (for a review see Schellenberg, 2001). Research into beneficial long-term effects of listening to music on physiological and psychological functioning has largely been neglected, with a few notable exceptions. In animals, for instance, repeated music exposure significantly altered neurotrophin levels in the hypothalamus (Angelucci, Ricci, Padua, Sabino, \& Tonali, 2007), which may alter hypothalamus-pituitary-adrenal axis activation. In humans, repeated music listening resulted in the following significant benefits: less depressed and confused mood in patients after stroke (Särkämö et al., 2008), reductions in high systolic blood pressure (Teng, Wong, \& Zhang, 2007), better quality of sleep in elderly patients (Lai \& Good, 2005) and reduced depression, mood disturbances, fatigue, and decreases in cortisol concentration in healthy adults (McKinney, Antoni, Kumar, Tims, \& McCabe, 1997). Repeated everyday listening to music may therefore also have a beneficial long-term effect.

Barely any attempts have been undertaken to reveal the underlying mechanisms or variables that mediate the effect of repeated music listening on various aspects of physiological and psychological functioning. Mitchell, MacDonald, Knussen, and Serpell (2007) conducted a cross-sectional survey with chronic pain sufferers in which the authors assessed music-listening behaviour and quality of life (QOL) as well as pain levels. Their findings indicate that specific aspects of music-listening behaviour, such as the average time per day spent listening to music, subjective relevance of music and reasons for listening to music (RML), may play an essential role. The latter were all concerned with pain-related coping (e.g. 'to help pain', 'to relieve tension and stress', 'to feel relaxed', 'to express emotions'). However, it is to be expected that a variety of other reasons for listening are relevant in a non-patient sample. For example, in the sample by North and colleagues, healthy adolescents' reasons for listening differed quite widely, e.g. 'to relieve boredom', 'to help me through difficult times', 'to please friends', 'to reduce loneliness' (North, Hargreaves, \& O'Neill, 2000). However, these authors did not examine associations between reasons for listening and indicators for physiological and psychological functioning in their participants. Thus, to the best of our knowledge, there are no data regarding the relationship between RML and indicators for physiological and psychological functioning in healthy adults.

Moreover, Mitchell et al. (2007) provided no explanation regarding the underlying mechanisms that may mediate the purported association between reasons 
for music listening and physiological and psychological functioning. Previous research indicates that music listening is extensively used for emotion regulation purposes (Juslin \& Sloboda, 2010; Saarikallio \& Erikkilä, 2007; Thoma, Ryf, Ehlert, $\&$ Nater, 2006). Emotion regulation is defined as '.. a process by which individuals influence which emotions they have, when they have them, and how they experience and express these emotions' (Gross, 1998, p. 275). It has been found that individuals differ in their habitual way to regulate their emotions (i.e. dispositional emotion regulation; Mohiyeddini, 2005; see also Wirtz et al., 2006). What is more, it has been reported that emotion regulation is linked to psychological and physiological functioning (Lam, Dickerson, Zoccola, \& Zaldivar, 2009). However, potential mediating effects of emotion regulation processes on the association between listening to music and physiological and psychological functioning have not been described in the literature so far. RML have also been related to stress-reducing purposes (Thayer, Newman, \& McCain, 1994). The term 'stress reduction' refers to techniques or strategies that aim to decrease the experience of stress which occurs whenever situational demands tax or exceed the resources of an afflicted individual (Lazarus \& Folkman, 1984). As per emotion regulation, individuals differ with regard to their general reactivity to stress (Schulz, Jansen, \& Schlotz, 2005), which has been related to psychological and physiological functioning. Therefore, it might be assumed that, depending on how individuals habitually respond to stressors (i.e. individual stress reactivity), people's RML, and consequently, its effect on physiological and psychological functioning, may differ. However, as with emotion regulation, no mediator model has tested an assumed mediating effect of individual stress reactivity on the association between listening to music and physiological and psychological functioning.

In the current research literature on the beneficial effect of listening to music, we identified a lack of studies investigating associations between various aspects of habitual music-listening behaviour and physiological and psychological functioning in adults. Potential mediating mechanisms, such as emotion regulation processes or individual stress reactivity, have not yet been examined. We therefore set out to investigate the interrelation between various aspects of habitual music-listening behaviour and physiological and psychological functioning, and the potential mediating influence of emotion regulation processes and individual stress reactivity.

\section{Method}

\section{Participants and data collection procedure}

A large online survey in a student population at the University of Zurich and the Zurich Federal Institute of Technology was conducted. Both institutions provided electronic mailing lists of students and faculty members who had previously provided permission of being contacted for research purposes. The email recipients were sent an invitation email with the invitation to enrol online for a survey about music, body and emotion. In the case of interest, the potential participants had access to the survey via an anonymous internet link embedded in the email. In order to ensure higher participation rates (Bosnjak \& Tuten, 2003), six cinema tickets and a dining coupon were raffled off. The survey was programmed online using the software Unipark (Unipark, Germany). For statistical analysis, the collected data were exported into SPSS 17.0 (SPSS, Chicago, Illinois). In a pilot study, a minimum time 
requirement of $25 \mathrm{~min}$ to complete the questionnaire was determined; participants who answered all questions substantially more quickly than others were excluded from the data set. All participants took part in the survey voluntarily and provided informed consent. The study was approved by the Swiss ethics committee of the Canton of Zurich.

\section{Materials}

The complete online survey consisted of 292 items. The first section consisted of the informed consent and questions addressing sociodemographic and general healthrelated variables. Participants who did not provide informed consent were unable to continue with the questionnaire and automatically directed to exit the questionnaire. The second section comprised questions about habitual music-listening behaviour (average duration of music listening per day, reasons and motivations for listening to music (RML), preferred music styles and subjective relevance of music), emotion regulation (dispositional emotion regulation), stress variables (dispositional stress reactivity) and health variables (somatic complaints and QOL). The following questionnaires were presented in random order.

\section{Music-listening behaviour and music preference}

The Music Preference Questionnaire (Nater, Krebs, \& Ehlert, 2005) was used to assess music-listening behaviour in terms of subjective importance of music, time spent listening to music and RML, as well as general music preference. As RMLs, the following 10 options were given: to relax, be activated, be distracted, reduce aggression, reduce loneliness, arouse specific emotions, increase specific emotions, work better and avoid boredom. The respondents could additionally add their own reasons under 'others'.

\section{Physiological and psychological functioning}

In the current study, physiological and psychological functioning was operationalised by the German self-report 'Freiburg Complaint List' (Freiburger Beschwerdeliste, FBL; Fahrenberg, 1994) and a German version of the Quality of Life Questionnaire of the World Health Organization (WHOQoL-5, WHOQoL-Group, 1998a, b). The FBL is a well-validated inventory for different aspects of bodily complaints and normal functioning. The questionnaire consists of nine subscales (General Condition, Tiredness, Cardiovascular, Gastrointestinal, Head-Throat, Tenseness, Emotional Reactivity, Pain and Sensory) and a summary scale, which are derived from 80 items. Fifty-seven items, such as 'Do you get headaches?', have to be answered by indicating one of five frequency categories (almost everyday, about three times a week, about twice a month, about twice a year and almost never). Twenty-three items, such as 'Are you sensitive to pain?', have to be answered by indicating one of five intensity categories (very strongly, strongly, moderately, barely and insensitive). Higher scores on FBL scales indicate frequent and intense complaints and go along with a negative general well-being. The FBL has been standardised with a representative sample of $N=2070$ German women and men aged 16 and older (Fahrenberg, 1995). Reliability coefficients (Cronbach's alpha) for the subscales and the total score range between 0.73 and 0.90 . In terms of validity, 
the total score of the FBL has been shown to be substantially associated with frequency of visiting practitioners and of in-patient treatment over and above effects of gender, age and social class. Moreover, FBL scores were consistently higher for psychotherapy patients compared to controls (Fahrenberg, 1995).

The WHOQoL-instruments (WHOQoL-100 and WHOQoL-BREF, a 25 items version of the WHOQoL; Angermeyer, Kilian, \& Matschinger, 2000) generally assess participants' QOL '.... broad-ranging concept, incorporating in a complex way the person's physical health, psychological state, level of independence, social relationships, and their relationship to salient features of their environment' (WHOQoL-Group, 1994, p. 43). The WHOQoL-instruments have been standardised cross-culturally using samples from several countries among them a Germanspeaking version. The WHOQoL-instruments have been demonstrated to have good discriminate validity with regard to ill and well respondents; good content validity as well as internal consistency (Cronbach's alpha ranging from 0.66 to 0.84 for the subscales; e.g. WHOQoL; Group, 1998). In the current study, we applied the WHOQoL-5. The WHOQoL-5 is a short version of the WHOQoL-instruments and consists of five items covering overall life satisfaction. Participants are asked to answer the items with regard to the last 2 weeks. Six answer options range from 0 (at no time point) to 5 (the whole time). The WHOQoL-5 has not yet been tested for its psychometric measures in healthy participants, but has previously been applied in various different WHO projects (Biering-Sorensen et al., 2006).

\section{Emotion regulation}

The German Inventory for Regulation of Emotion (ERI, [Inventar zur Emotionsregulationswirksamkeit]; Mohiyeddini, 2005; see also Wirtz et al., 2006) was used to assess the dispositional regulation of emotions. The ERI has 34 items and comprises a hedonistic (HED) way of emotion regulation, a distress-augmenting regulation (DAR), i.e. intensifying of negative emotions, and an emotional moderation regulation (MOD), i.e. buffering of emotions. Using a 6-point Likert scale ranging from 1 (hardly ever) to 6 (almost always), the participants were asked to rate how often they use specific strategies (e.g. go out with friends, thinking of something cheerful) to regulate their emotions.

\section{Inter-individual stress reactivity}

The German Stress Reactivity Scale (SRS, [Stress-Reaktivitaets-Skala]; Schulz et al., 2005) was used to assess general susceptibility to stress, i.e. the disposition to respond to stressors with intense, immediate and long-lasting stress reactions. Four personality characteristics are assumed to determine stress reactivity: high intrusiveness, low self-efficacy, high arousability of the central nervous system and high negative affectivity. The SRS assesses general and specific stress reactivity in different stressor domains.

\section{Data analyses}

Data were analysed by means of regression analyses using path models with AMOS 17.0 (Arbuckle \& Wothke, 1999). Mediator hypotheses were tested according to recommendations by Baron and Kenny (1986). For each full model, three regression 
analyses were conducted. The first tested whether the predictor was significantly associated with the outcome. The second tested whether the predictor was significantly associated with possible mediators and the final analysis included the predictor and the mediator variables as predictors of the outcome. When the previously significant direct effect of the predictor on the outcome decreases to a non-significant level after the mediator variables are included, the effect can be regarded as fully mediated. Reduction in strength of the association can be seen as a partial mediation. Furthermore, the indirect effect from the predictor through the mediator on the outcome was tested for statistical significance (i.e. whether or not it is different from zero) using the Sobel test (Sobel, 1982).

Missing data were treated using the full information maximum likelihood (FIML) algorithm. FIML is a method that uses all available information of all observed (also incomplete) cases in order to enhance the validity of parameter estimation. FIML is superior to common methods such as listwise or pairwise deletion (Enders \& Bandalos, 2001) and was thus chosen for the present studies. Univariate and multivariate outliers were routinely screened and treated as suggested by Tabachnick and Fidell (2001).

Results

Participants' characteristics

A total of $N=22,179$ recipients were sent an invitation email of which $N=2616$ provided data sets. This is a response rate of $11.79 \%$. After cleaning the data pool, i.e. removing incomplete or incorrect data sets, and excluding data sets of participants who answered the questionnaire substantially faster than within 25 min, we obtained a final $N$ of $1230(24.89 \pm 5.34$ years old; 683 females $(55.3 \%)$ and 547 males) participants who completed all questionnaires. All participants were students or junior university staff members and were therefore highly comparable with regard to their level of education.

Four models were tested: the first mediator model specified RML reducing loneliness, the second RML reducing aggression as predictor, DAR and stress reactivity as mediators and QOL and sum scores of the FBL as outcomes. The third and fourth mediator models specified RML arousing specific feelings and RML intensifying specific feelings as predictors, DAR and stress reactivity as mediators and only the sum scores of the FBL as outcomes (a table of raw correlations between variables of interest is available upon request).

\section{Mediator model with RML reducing loneliness}

In a first model, we tested whether there were direct effects of RML reducing loneliness on QOL and the sum scores of the FBL when controlling for gender. Results are displayed in Figure 1. The substantial associations between RML reducing loneliness and QOL $(\beta=-0.21)$ and sum scores of the FBL $(\beta=0.24)$ remained when controlling for gender $(\beta=0.27$ for the association with the sum scores of the FBL, indicating higher reporting of symptoms of the FBL for women; $\beta=-0.01$, ns for the association with QOL). The definite mediator model included stress reactivity and DAR as mediator variables. Indirect effects between RML reducing loneliness via stress reactivity to QOL (Sobel $Z=-7.22, p=0.001$ ) and the 


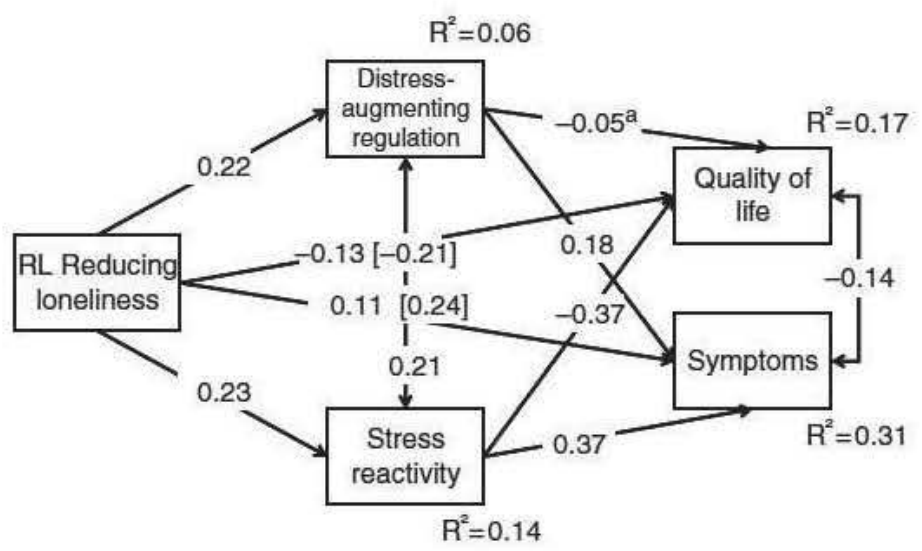

Figure 1. Mediator model with RML reducing loneliness.

Notes: Coefficients in parentheses: direct effects without mediator variables. All coefficients reported are controlled for gender. Associations with gender: $\beta \quad 0.09$, with DAR; $\beta \quad 0.29$ with stress reactivity; $\beta \quad 0.16$ with quality of life; $\beta \quad 0.14$ with sum scores of the FBL; $r \quad 0.05$ with RML reducing loneliness. Gender coded 1 men, 2 women. Unless otherwise indicated, all coefficients were significant at $p<0.01 ;{ }^{a} p<0.10$.

sum scores of the FBL (Sobel $Z=7.39, p=0.001$ ) as well as via DAR to sum scores of the FBL (Sobel $Z=5.30, p=0.001$ ) reached significance. The indirect effect between RML reducing loneliness via DAR to QOL (Sobel $Z=-1.82, p=0.07$ ) was not significant at the $5 \%$ level. Overall, due to the considerable reduction of the direct effects, the associations between RML reducing loneliness and QOL and sum scores of the FBL are mediated by stress reactivity and for values of sum scores of the FBL as outcomes also in part by DAR.

\section{Mediator model with RML reducing aggression}

In a second model, we tested whether there were direct effects of RML reducing aggression on QOL and sum scores of the FBL when controlling for gender. The associations between RML reducing aggression and QOL $(\beta=-0.15)$ and sum scores of the FBL $(\beta=0.17)$ remained almost unchanged when controlling for gender ( $\beta=0.27$ for the association with the sum scores of the FBL, indicating higher reporting of symptoms of the FBL for women; $\beta=-0.01$, ns for the association with QOL). The definite mediator model included stress reactivity and DAR as mediator variables. Results are displayed in Figure 2 and are comparable to RML reducing loneliness. Indirect effects between RML reducing aggression via stress reactivity to QOL (Sobel $Z=-4.37, p=0.001$ ) and sum scores of the FBL (Sobel $Z=4.41$, $p=0.001$ ) as well as via DAR to sum scores of the FBL (Sobel $Z=4.73, p=0.001$ ) reached statistical significance. The indirect effect between RML reducing aggression via DAR to QOL (Sobel $Z=-1.95, p=0.05$ ) was not significant at the $5 \%$ level. Overall, due to the considerable reduction of the direct effects, the associations between RML reducing aggression and QOL and sum scores of the FBL were mediated by stress reactivity and for sum scores of the FBL as outcomes also by DAR. 


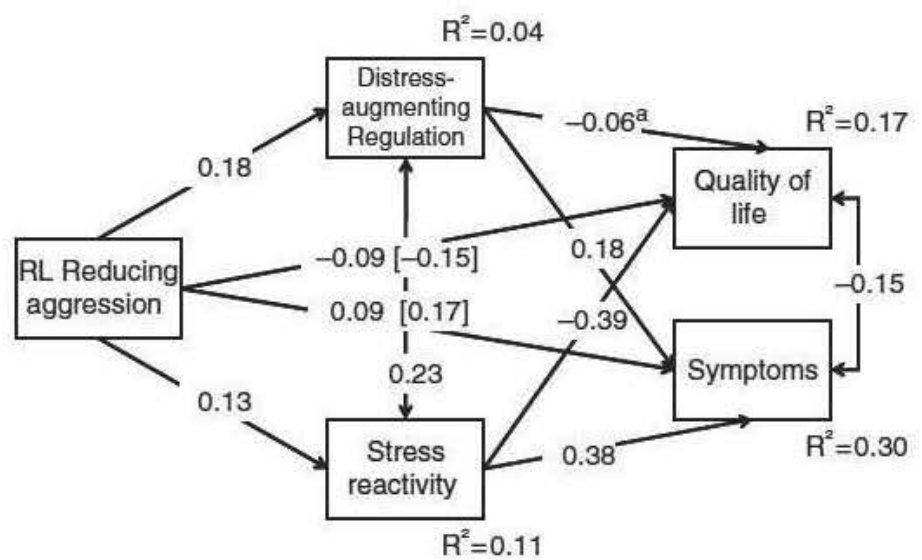

Figure 2. Mediator model with RML reducing aggression.

Notes: Coefficients in parentheses: direct effects without mediator variables. All coefficients reported are controlled for gender. Associations with gender: $\beta \quad 0.10$, with DAR; $\beta \quad 0.29$ with stress reactivity; $\beta \quad 0.11$ with quality of life; $\beta \quad 0.14$ with sum scores of the FBL; $r 0.05, p<0.10$ with RML reducing aggression. Gender coded 1 men, 2 women. Unless otherwise indicated, all coefficients were significant at $p<0.01 ;{ }^{a} p<0.05$.

\section{Mediator model with RML arousing specific feelings}

In a third model, we tested whether there were direct effects of RML arousing specific feelings on the sum scores of the FBL when controlling for gender. The associations between RML arousing specific feelings and sum scores of the FBL $(\beta=0.16)$ remained unchanged when controlling for gender $(\beta=0.28$ for the association with the sum scores of the FBL, indicating higher reporting of symptoms in the FBL for women; $\beta=-0.01$ ). The definite mediator model included stress reactivity and DAR as mediator variables. Results are displayed in Figure 3. Both indirect effects between RML arousing specific feelings via stress reactivity to sum scores of the FBL (Sobel $Z=3.54, p=0.001$ ) as well as via DAR to sum scores of the FBL (Sobel $Z=5.69, p=0.001$ ) reached significance. Overall, due to the reduction of the direct effect, the association between RML arousing specific feelings and sum scores of the FBL was mediated by stress reactivity and by DAR.

\section{Mediator model with RML intensifying specific feelings}

In a fourth model, we tested whether there were direct effects of RML intensifying specific feelings on sum scores of the FBL when controlling for gender. The associations between RML intensifying specific feelings and sum scores of the FBL ( $\beta=0.19)$ remained unchanged when controlling for gender $(\beta=0.29$ for the association with sum scores of the FBL, indicating higher reporting of symptoms in the FBL for women). The definite mediator model included stress reactivity and DAR as mediator variables. As results are very similar to those for RML arousing specific feelings, no additional figure is provided. RML intensifying specific feelings was associated with $\beta=0.10(p=0.001)$ to stress reactivity and with $\beta=0.32$ $(p=0.001)$ with DAR. Stress reactivity and DAR, in turn, were significantly related 


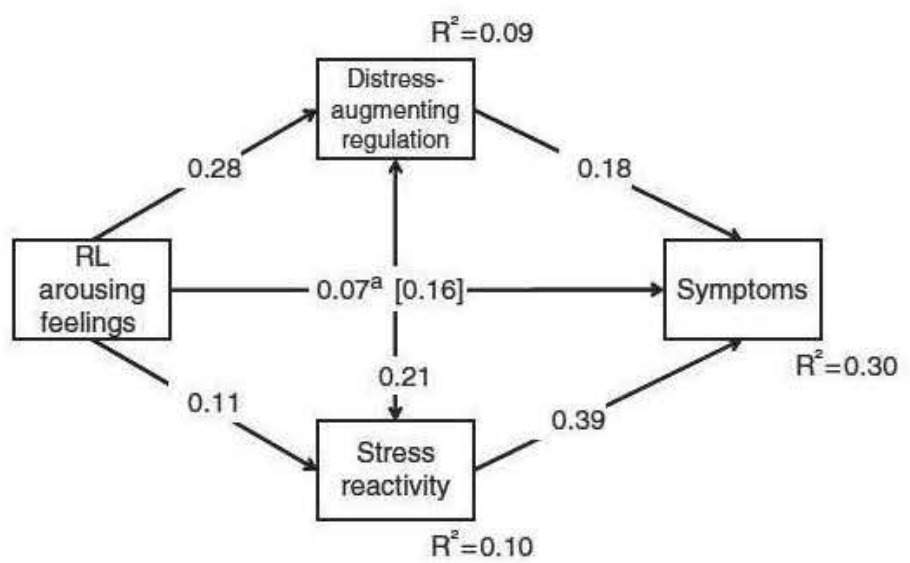

Figure 3. Mediator model with RML arousing specific feelings.

Notes: Coefficient in parentheses: direct effect without mediator variables. All coefficients reported are controlled for gender. Associations with gender: $\beta \quad 0.11$, with DAR; $\beta \quad 0.30$ with stress reactivity; $\beta \quad 0.14$ with sum scores of the FBL; $r \quad 0.01$, ns with RML arousing specific feelings. Gender coded 1 men, 2 women. Unless otherwise indicated, all coeffi cients were significant at $p<0.01 ;{ }^{\mathrm{a}} p<0.05$.

to sum scores of the FBL $(\beta=0.39, p=0.001$ for stress reactivity and $\beta=0.16$, $p=0.001$ for DAR). Both indirect effects between RML intensifying specific feelings via stress reactivity to sum scores of the FBL (Sobel $Z=3.39, p=0.001$ ) as well as via DAR to sum scores of the FBL (Sobel $Z=5.51, p=0.001$ ) reached significance. Overall, due to the reduction of the direct effect, the association between RML intensifying specific feelings and sum scores of the FBL was mediated by stress reactivity and by DAR.

\section{Discussion}

This study examined the interrelation between habitual music-listening behaviour and physiological and psychological functioning. We hypothesised that emotion regulation processes and individual stress reactivity accounted for potential associations between habitual music-listening behaviour and indicators of physiological and psychological functioning. Our findings indicate that average duration of music listening or subjective relevance of music are not associated with physiological and psychological functioning. In contrast, RML (especially 'reducing loneliness and aggression' and 'arousing or intensifying specific emotions') seem to play a much more crucial role. These effects were mediated by distress-augmenting emotion regulation and individual stress reactivity.

It appears that in non-patient adults, qualitative aspects of listening to music, i.e. reasons related to emotion regulation, are more important for the general physiological and psychological functioning than quantitative aspects, i.e. average duration of music listening or subjective relevance of music. This result is in contrast to findings by Mitchell et al. (2007), who found that both frequent music listening and subjective importance of music were related to higher QOL and lesser need for medical treatment in pain sufferers. In our study, the mean ratings of subjective 
relevance of music were rather high in most participants. Consequently, the fact that we did not find an interrelation between the subjective relevance of music and indicators of physiological and psychological functioning may be due to a ceiling effect. Moreover, given the particular characteristics of the sample by Mitchell et al. (2007), i.e. chronic pain sufferers, comparisons with our sample, i.e. non-patient adults, are limited. Differences in the basic health status of the two samples are probably the main reason for the identified discrepancies between the results of the two studies.

We found a direct negative association between the reason for listening to reduce loneliness' and indicators of physiological and psychological functioning (i.e. negative association with QOL and positive association with number of somatic symptoms). Other studies examining chronic pain sufferers (Mitchell et al., 2007) and adolescents (North et al., 2000) also reported that reducing loneliness was a major reason for listening to music. However, the negative association with indicators of physiological and psychological functioning has not yet been documented in the literature. Although we did not measure loneliness per se, we might assume that those respondents who reported listening to music in order to reduce loneliness may also experience this feeling more often. One experiences the distressing feeling of loneliness in times when one's own social contacts are perceived as less fulfilling than one whishes them to be (Peplau \& Perlman, 1982). Loneliness and its related social isolation have been associated with increased physical health risks (Cole, 2008), reduced longevity (Peel, McClure, \& Bartlett, 2005), increased risk of cancer (Reynolds \& Kaplan, 1990), increased depressive symptoms (Cacioppo \& Hawkley, 2003) and poor lifestyle behaviour, such as smoking, physical inactivity and overweight (Hawkley, Thisted, \& Cacioppo, 2009; Lauder, Mummery, Jones, \& Caperchione, 2006). Our finding of a negative association between the reason of listening to reduce loneliness, as an indirect indicator of loneliness, and indicators of physiological and psychological functioning is therefore in line with previous studies relating to the topic of ill health through loneliness.

Further, we found a direct negative association between the reason for listening 'to reduce aggression' and indicators of physiological and psychological functioning (i.e. negative association with QOL and positive association with number of somatic symptoms). This is a novel finding, which has not been reported previously. As with loneliness, we did not measure aggression as such, but we may assume that those respondents reporting to listening to music in order to reduce (feelings or behavioural tendencies associated with) aggression may also experience it more often. Aggression describes a behaviour that is performed to injure or hurt somebody who avoids being a target of these actions (Baron \& Richardson, 2004). It has previously been associated with several health issues, such as depression and chronic stress, or poor lifestyle behaviour, such as smoking and drinking (Johnson, 1990). Our finding of a negative association between the reason of listening to reduce aggression (as an indirect indicator of aggression) and indicators of physiological and psychological functioning is therefore consistent with previous findings relating to the topic of ill health through aggression.

Finally, we found a direct negative association between the reasons for listening 'arousing and intensifying specific feelings' and indicators of physiological and psychological functioning (i.e. positive association with number of somatic symptoms). This is in line with previous studies (Mitchell et al., 2007; North \& Hargreaves, 2000). The emotion-eliciting capacity of music is considered to be the 
main reason for actively engaging with music (Juslin \& Sloboda, 2001). Accordingly, our finding appears rather surprising and raises new questions. This observation can only be interpreted when the mediating variables, i.e. DAR and individual stress reactivity, are taken into account. DAR describes the disposition of an individual to intensify negative emotions. It is therefore assumed that individuals who have the disposition to intensify negative emotions also listen to music with the purpose of arousing or intensifying negative emotions. Previous research indicates that negative emotions are associated with negative health outcomes (Kubzansky \& Kawachi, 2000). Our finding is therefore consistent with results of previous investigations. The same explanation might also be valid for the second mediating variable, i.e. stress reactivity. The construct of stress reactivity describes the disposition of an individual to respond to stressors with immediate, strong and long-term stress reactions (Schulz et al., 2005). We therefore assume that respondents with this disposition also experience emotions associated with stress more often (such as anxiety or anger) and consequently preferentially listen to music with the purpose of arousing or intensifying these emotions. Sustained stress experience and its biological consequences negatively impact psychological and physiological health (Esch, Stefano, Fricchione, \& Benson, 2002). Thus, the negative association between the reason for listening 'arousing and intensifying specific feelings' and indicators of physiological and psychological functioning can be explained by the mediating influence of stress reactivity.

Does listening to music improve physiological and psychological functioning? Our findings indicate that it might not be possible to answer this question with a simple yes or no. Habitual music-listening behaviour appears to be a multifaceted behaviour that consists of many different aspects. In addition, the use of music seems to be further influenced by individual dispositions (emotion regulation and susceptibility to stress). Moreover, it is likely that use and subsequent effects of music listening are further influenced by other variables, such as personality factors (Chamorro-Premuzic, Swami, Furnham, \& Maakip, 2009). Finally, physiological and psychological functioning is also further influenced by various habitual behaviours, such as physical exercise (Waddington, Malcolm, \& Green, 1997) or diet (Cordain et al., 2005). From this perspective, the fact that we were still able to detect some associations between habitual music-listening behaviour and indicators of physiological and psychological functioning further emphasises the relevance of this topic. However, to achieve a better understanding of this association, future studies should also include the above-mentioned influencing factors.

There are several limitations to this study that should be acknowledged.

Survey respondents: As we were studying the associations between music listening and indicators of physiological and psychological functioning in a wide university population, the generalisation of our results beyond this sample is restricted. It is to be assumed that participants in our sample, consisting mainly of university students, have different everyday lives than other samples, and might therefore show different music-listening behaviour. Moreover, as respondents were aware of the topic of the survey, i.e. music, and due to the voluntary nature of study participation, a selection bias may have confounded our results. The high amount of average time spent listening to music and the high values of the subjective relevance of music are indicators of this assumption. Consequently, the findings should be replicated in a 
larger and more heterogeneous sample consisting of respondents who are more naïve to the intentions of the survey.

Setting: A web-based data collection has many advantages: besides its convenience and anonymity for respondents, it is cost-effective and allows access to a broad and homogeneous sample as well as the assessment of data in a short period of time. The shortcomings of this approach are the assumed biased, i.e. non-random response and the generally lower response rates in comparison to conventional paper-and-pencil methodology (for a review see Cook, Heath, \& Thompson, 2000).

Cross-sectional design: Given the fact that our survey was a cross-sectional study, we are only able to reveal associations between the variables of interest and unable to establish any causal interpretations between them. Only a broadly constructed prospective study design, i.e. a longitudinal correlational study, which controls for a multitude of influencing variables, might provide a comprehensive answer to the question of whether listening to music in everyday life does indeed have a positive long-term impact on health.

In conclusion, this was the first study to investigate the associations between music-listening behaviour and indicators of physiological and psychological functioning in a sample of adult non-patients. We were able to demonstrate that qualitative aspects of music-listening behaviour, i.e. specific (negative) emotionregulated reasons for listening, are directly associated with indicators of physiological and psychological functioning. We also found that these associations are mediated by dispositional emotion regulation processes and individual stress reactivity. Thus, individuals use music differentially, depending on their habitual way of regulating their emotions and on their individual susceptibility to stress. Based on this knowledge, music listening might be more systematically used in therapeutic settings, thus enhancing its clinical relevance.

\section{Acknowledgements}

This study was supported by a grant from the Young Investigator Grant of the University of Zurich. Grant no: 56233208 (MVT). We thank the survey respondents and acknowledge the assistance of Martial Böni and Regula Flütsch. We would like to thank Prof. Changiz Mohiyeddini for providing us with the ERI. UMN acknowledges support by the Volkswagen Foundation.

\section{References}

Angelucci, F., Ricci, E., Padua, L., Sabino, A., \& Tonali, P.A. (2007). Music exposure differentially alters the levels of brain derived neurotrophic factor and nerve growth factor in the mouse hypothalamus. Neuroscience Letters, 429, 152 155, doi:10.1016/ j.neulet.2007.1010.1005.

Angermeyer, M.C., Kilian, R., \& Matschinger, H. (2000). WHOQOL 100 und WHOQOL BREF: Handbuch für die deutsche Version der WHO Instrumente zur Erfassung von Lebensqualität. Göttingen: Hogrefe.

Arbuckle, J.L., \& Wothke, W. (1999). Amos (version 4.0). Chicago: Smallwaters. 
Baron, R.M., \& Kenny, D.A. (1986). The mediator moderator variable distinction in social psychological research: Conceptual, strategic, and statistical considerations. Journal of Personality and Social Psychology, 51, 11731182.

Baron, R.A., \& Richardson, D.R. (2004). Human aggression (2nd ed.). New York, NY: Plenum.

Biering Sorensen, F., Scheuringer, M., Baumberger, M., Charlifue, S.W., Post, M.W., Montero, F., \& Stucki, G. (2006). Developing core sets for persons with spinal cord injuries based on the international classification of functioning, disability and health as a way to specify functioning. Spinal Cord, 44, 541546.

Blood, A.J., \& Zatorre, R.J. (2001). Intensely pleasurable responses to music correlate with activity in brain regions implicated in reward and emotion. Proceedings of the National Academy of Sciences of the United States of America, 98, 1181811823.

Bosnjak, M., \& Tuten, T.L. (2003). Prepaid and promised incentives in web surveys: An experiment. Social Science Computer Review, 21, 208217.

Burns, J.L., Labbe, E., Arke, B., Capeless, K., Cooksey, B., Steadman, A., \& Gonzales, C. (2002). The effects of different types of music on perceived and physiological measures of stress. Journal of Music Therapy, 39, 101116.

Cacioppo, J.T., \& Hawkley, L.C. (2003). Social isolation and health, with an emphasis on underlying mechanisms. Perspectives in Biology and Medicine, 46, 3952.

Chamorro Premuzic, T., Swami, V., Furnham, A., \& Maakip, I. (2009). The big five personality traits and uses of music. Journal of Individual Differences, 30, 2027.

Cole, W.C. (2008). Social regulation of leukocyte homeostasis: The role of glucocorticoid sensitivity. Brain, Behavior, and Immunity, 22, 10491055.

Cook, C., Heath, F., \& Thompson, R.L. (2000). A meta analysis of response rates in web or internet based surveys. Education and Psychological Measurement, 60, 821836.

Cordain, L., Eaton, S.B., Sevastian, A., Mann, N., Lindeberg, S., Watkins, B.A., \& Brand Miller, J. (2005). Origins and evolution of the Western diet: Health implications for the 21th century. American Journal of Clinical Nutrition, 81, 341354.

Ebneshahidi, A., \& Mohseni, M. (2008). The effect of patient selected music on early postoperative pain, anxiety, and hemodynamic profile in cesarean section surgery. Journal of Alternative Complementary Medicine, 14, 827831.

Enders, C.K., \& Bandalos, D.L. (2001). The relative performance of full information maximum likelihood estimation for missing data in structural equation models. Structural Equation Modeling, 8, 430457.

Esch, T., Stefano, G.B., Fricchione, G.L., \& Benson, H. (2002). An overview of stress and its impact in immunological diseases. Modern Aspects of Immunobiology, 2, 187192.

Evans, D. (2002). The effectiveness of music as an intervention for hospital patients: A systematic review. Journal of Advanced Nursing, 37, 818.

Fahrenberg, J. (1994). Die freiburger beschwerdenliste (FBL): Form FBL G und revidierte form FBL R. Göttingen: Hogrefe.

Fahrenberg, J. (1995). Somatic complaints in the German population. Journal of Psychosomatic Research, 39, 809817.

Gross, J.J. (1998). The emerging field of emotion regulation: An integrative review. Review of General Psychology, 2, 271299.

Group, T.W. (1998). The World Health Organization Quality of Life Assessment (WHOQoL): Development and general psychometric properties. Social Science and Medicine, 46, 15691585.

Hawkley, L.C., Thisted, R.A., \& Cacioppo, J.T. (2009). Loneliness predicts reduced physical activity: Cross sectional \& longitudinal analysis. Health Psychology, 28, 354363.

Johnson, E.H. (1990). The deadly emotions: The role of anger, hostility, and aggression in health and emotional well being. New York, NY, England: Praeger.

Juslin, P.N., \& Sloboda, J.A. (2001). Music and emotion: Theory and research. London: Oxford University Press. 
Juslin, P.N., \& Sloboda, J. (2010). Handbook of music and emotion. New York, NY: Oxford University Press.

Khalfa, S., Bella, S.D., Roy, M., Peretz, I., \& Lupien, S.J. (2003). Effects of relaxing music on salivary cortisol level after psychological stress. Annals of the New York Academy of Sciences, 999, 374376.

Kubzansky, L.D., \& Kawachi, I. (2000). Going to the heart of the matter: Do negative emotions cause coronary heart disease? Journal of Psychosomatic Research, 48, 323337.

Lai, H.L., \& Good, M. (2005). Music improves sleep quality in older adults. Journal of Advanced Nursing, 49, 234244.

Lam, S., Dickerson, S.S., Zoccola, P.M., \& Zaldivar, F. (2009). Emotion regulation and cortisol reactivity to a social evaluative speech task. Psychoneuroendocrinology, 34, 13551362.

Lauder, W., Mummery, K., Jones, M., \& Caperchione, C. (2006). A comparison of health behaviours in lonely and non lonely populations. Psychology, Health and Medicine, 11, 233245.

Lazarus, R.S., \& Folkman, S. (1984). Stress, appraisal, and coping. New York, NY: Springer.

McKinney, C.H., Antoni, M.H., Kumar, M., Tims, F.C., \& McCabe, P.M. (1997). Effects of guided imagery and music (GIM) therapy on mood and cortisol in healthy adults. Health Psychology, 16, 390400.

Mitchell, L.A., MacDonald, R.A.R., Knussen, C., \& Serpell, M.G. (2007). A survey investigation of the effects of music listening on chronic pain. Psychology of Music, 35, 3757.

Mohiyeddini, C. (2005). Personality, emotion regulation and psychopathology.University of Tuebingen, Germany.

Nater, U.M., Abbruzzese, E., Krebs, M., \& Ehlert, U. (2006). Sex differences in emotional and psychophysiological responses to musical stimuli. International Journal of Psychophysiology, 62, 300308.

Nater, U.M., Krebs, M., \& Ehlert, U. (2005). Sensation seeking, music preference and psychophysiological reactivity to music. Musicae Scientiae, 9, 239254.

Nilsson, U. (2008). The anxiety and pain reducing effects of music interventions: A systematic review. AORN Journal, 87, 780807.

North, A.C., \& Hargreaves, D.J. (2000). Musical preferences during and after relaxation and exercise. American Journal of Psychology, 113, 4367.

North, A.C., Hargreaves, D.J., \& O’Neill, S.A. (2000). The importance of music to adolescents. The British Journal of Educational Psychology, 70, 255272.

Peel, N.M., McClure, R.J., \& Bartlett, H.P. (2005). Behavioral determinants of healthy aging. American Journal of Preventive Medicine, 28, 298304.

Peplau, L.A., \& Perlman, D. (1982). Perspectives on loneliness. In L.A. Peplau \& D. Perlman (Eds.), Loneliness: A sourcebook of current theory, research and therapy (pp. 1 20). New York, NY: Wiley.

Pothoulaki, M., MacDonald, R.A.R., Flowers, P., Stamataki, E., Filiopoulos, V., Stamatiadis, D., \& Stathakis, Ch.P. (2008). An investigation of the effects of music on anxiety and pain perception in patients undergoing haemodialysis treatment. Journal of Health Psychology, 13, 912920.

Reynolds, P., \& Kaplan, G.A. (1990). Social connections and risk for cancer: Prospective evidence from the Alameda County Study. Behavioral Medicine, 16, 101110.

Roy, M., Peretz, I., \& Rainville, P. (2007). Emotional valence contributes to music induced analgesia. Pain, 134, 140147.

Saarikallio, S., \& Erikkilä, J. (2007). The role of music in adolescents' mood regulation. Psychology of Music, 35, 88109.

Särkämö, T., Tervaniemi, M., Laitinen, S., Forsblom, A., Soinila, S., Mikkonen, M., Autti, T., \& Hietanen, M. (2008). Music listening enhances cognitive recovery and mood after middle cerebral artery stroke. Brain, 131, 866876. 
Schellenberg, E.G. (2001). Music and nonmusical abilities. Annals of the New York Academy of Sciences, 930, 355371

Schulz, P., Jansen, L., \& Schlotz, W. (2005). Stressreaktivität: Theoretisches Konzept und Messung. [Stress reactivity: Theoretical concept and measurement]. Diagnostica, 51, 124133.

Sobel, M.E. (1982). Asymptotic intervals for indirect effects in structural equations models. In S. Leinhart (Ed.), Socio meth (Vol. 1982, pp. 290 312). San Francisco, CA: Jossey Bass.

Tabachnick, B.G., \& Fidell, L.S. (2001). Using multivariate statistics (4th ed.). Boston, MA: Allyn and Bacon.

Teng, X.F., Wong, M.Y.M., \& Zhang, Y.T. (2007). The effect on music on hypertensive patients. In Proceedings of the 29th Annual International Conference of the IEEE EMBS (pp. 4649 4651). Lyon: Chinese University of Hong Kong. doi:10.1109/ IEMBS.2007.4353376.

Thayer, R.E., Newman, J.R., \& McCain, T.C. (1994). Self regulation of mood: Strategies for changing a bad mood, raising energy and reducing tension. Journal of Personality and Social Psychology, 67, 910925.

Thoma, M.V., Ryf, S., Ehlert, U., \& Nater, U.M. (2006). Regulation of emotions by listening to music in emotional situations. In M. Baroni, A.R. Addessi, R. Caterina, \& M. Costa (Eds.), Proceedings of the 9th International Conference on Music Perception and Cognition. Italy: University of Bologana.

Voss, J.A., Good, M., Yates, B., Baun, M.M., Thompson, A., \& Hertzog, M. (2004). Sedative music reduces anxiety and pain during chair rest after open heart surgery. Pain, 112, 197203

Waddington, I., Malcolm, D., \& Green, K. (1997). Sport, health and physical education: A reconsideration. European Physical Education Review, 3, 165182.

WHOQoL Group (1994). The development of the World Health Organization Quality of Life Assessment Instrument (WHOQOL). In J. Orley \& W. Kuyken (Eds.), Quality of life assessment: International perspectives. Heidelberg: Springer Verlag.

WHOQoL Group (1998a). Development of the World Health Organization WHOQOL BREF quality of life assessment. The WHOQOL Group. Psychological Medicine, 28, 551558.

WHOQoL Group (1998b). The World Health Organization Quality of Life Assessment (WHOQOL): Development and general psychometric properties. Social Science and Medicine, 46, 15691585.

Wirtz, P., Von Kanel, R., Mohiyeddini, C., Emini, L., Ruedisueli, K., Groessbauer, S., \& Ehlert, U. (2006). Low social support and poor emotion regulation are associated with increased stress hormone reactivity to mental stress in systemic hypertension. Journal of Clinical Endocrinology and Metabolism, 91, 38573865. 